\title{
LA EVALUACIÓN FORMATIVA Y COMPARTIDA EN EDUCACIÓN INFANTIL. CONSECUENCIAS DEL USO DE DOS METODOLOGÍAS DIFERENTES
}

Formative and Shared assessment in Kindergarten education. Consequences about using two different methods.

Avaliação formativa e partilhada na educação pré-escolar. Consequências da utilização de duas metodologias diferentes.

\section{Carla Fernández Garcimartín (1) \\ Víctor Manuel López Pastor (2) \\ Cristina Pascual Arias (3)}

\begin{abstract}
Apoyo/financiación del trabajo: Proyecto de Innovación Docente (PID) "La evaluación formativa y compartida en educación. Transferencia de conocimiento entre Universidad y Escuela". Financiado por la Universidad de Valladolid.

(1) Facultad de Educación de Segovia (Universidad Valladolid, España). Teléfono: +34 692533275. Correo electrónico: carlafdzg96@gmail.com

(2) Facultad de Educación de Segovia (Universidad Valladolid, España). Teléfono: +34 987293099. Correo electrónico: vlopez@mpc.uva.es

(3) Facultad de Educación de Segovia (Universidad Valladolid, España). Teléfono: +34 987293099. Correo electrónico: cristinapascuari@gmail.com
\end{abstract}

\section{Resumen}

Este trabajo muestra una experiencia de aplicación de un sistema de evaluación formativa y compartida en un aula de segundo de educación infantil. Se ha llevado a cabo durante el Prácticum II del grado de educación infantil, con una duración de tres meses. Se han realizado dos intervenciones en el aula, cada una con una metodología diferente. La experiencia se analizará para comprobar en qué tipo de metodología es más coherente en la aplicación de la evaluación formativa y compartida. A la hora de desarrollar este sistema de evaluación se han utilizado las siguientes técnicas e instrumentos de obtención de datos: (a) técnicas: observación participante, evaluación entre iguales, reflexión docente, evaluación compartida y entrevistas individuales; (b) instrumentos: cuaderno del profesor, fichas de seguimiento grupal, ficha de evaluación entre iguales, diario del profesor, rúbrica de aprendizaje y escala graduada. En el congreso se presentarán los resultados totales encontrados.

La evaluación formativa y compartida en Educación Infantil. Consecuencias del uso de dos metodologías 
Palabras clave: Evaluación formativa; evaluación compartida; educación infantil; metodologías didácticas; técnicas e instrumentos de evaluación

\begin{abstract}
This paper shows an experience of formative and shared assessment in a class of the second year of kindergarten education. The experience is carried out during the Practicum II of the bachelor's degree in kindergarten Education that lasts three months. Two interventions were carried out during the class: each one with different methods. The experience intends to verify in what type of methodology the application of the formative and shared assessment is more coherent. At the time of developing this assessment system, we have used the following techniques and data collection instruments: (a) techniques: participant observation, peer assessment, teacher reflection, shared assessment and individual interviews; (b) instruments: teacher's notebook, group tracking form, co-assessment form, teacher's diary, learning rubric and graded scale. The final results will be presented during the congress.
\end{abstract}

Keywords: Formative assessment; shared assessment; kindergarten education; didactic methodologies; assessment instruments and techniques

\title{
Resumo
}

Este trabalho mostra uma experiência de aplicação de um sistema de avaliação formativa e partilhada numa aula do segundo ano da educação pré-escolar. A experiência é levada a cabo durante a disciplina "Prática II" da licenciatura em educação pré-escolar, durante três meses. Realizaram-se duas intervenções durante a aula: cada uma com uma metodologia diferente. O objetivo desta experiência foi ver em que tipo de metodologia a aplicação da avaliação formativa e partilhada é mais coerente. No momento de desenvolver este sistema de avaliação, foram utilizadas as seguintes técnicas e instrumentos de recolha de dados: (a) Técnicas: observação participante, avaliação por pares, reflexão de professores, avaliação partilhada e entrevistas individuais; (b) Instrumentos: caderno do professor, ficha de acompanhamento de grupo, ficha de avaliação de pares, diário do professor, rubrica de aprendizagem e escala graduada. No congresso serão apresentados os resultados finais.

Palavras-chave: Avaliação formativa; avaliação partilhada; educação pré-escolar; metodologias didáticas; técnicas e instrumentos de avaliação

La evaluación formativa y compartida en Educación Infantil. Consecuencias del uso de dos metodologías diferentes 


\section{Introducción}

Presentamos una experiencia en educación infantil aplicando sistemas de Evaluación Formativa y Compartida (EFyC) en dos metodologías de trabajo diferentes. Se realiza durante el periodo de prácticas $\left(5^{\circ}\right.$ curso de doble grado de magisterio, febrero-mayo2019).

López-Pastor y Pérez-Pueyo (2017) definen la evaluación formativa como "todo proceso de evaluación cuya finalidad principal es mejorar los procesos de enseñanzaaprendizaje que tienen lugar" (p. 37). Esta alternativa a la evaluación tradicional tiene sentido cuando hablamos de Evaluación Compartida: "procesos de diálogo que mantiene el profesor con su alumnado sobre la evaluación de los aprendizajes y los procesos de enseñanza-aprendizaje que tienen lugar" (López-Pastor y Pérez-Pueyo, 2017, p. 45).

En esta experiencia educativa, los aprendizajes están adaptados a los intereses de los alumnos. Esta personalización del aprendizaje se refleja en la EFyC: "la evaluación debe estar, siempre y en todos los casos, al servicio de quienes son los protagonistas en el proceso de enseñanza y de aprendizaje, y especialmente al servicio de los sujetos que aprenden" (Álvarez-Méndez, 2001, p. 3).

\section{Contextualización}

La experiencia educativa se ha realizado en el CEIP Fray Juan de la Cruz, ubicado en pleno casco histórico de Segovia, que acoge a 204 alumnos. La experiencia se lleva a cabo con $2^{\circ}$ de Educación Infantil (23 alumnos). Es un grupo muy activo, formado mayoritariamente por chicos. Contamos con dos alumnos con necesidades educativas especiales (ACNEE): un alumno autista y un alumno con una discapacidad motórica izquierda (pierna y brazo). Ambos tienen ritmos de aprendizaje muy diferentes, necesitan adaptaciones y trabajo individualizado.

\section{Diseño y desarrollo}

El sistema de EFyC se lleva a cabo con dos intervenciones didácticas metodológicamente diferentes:

1- Partiendo de la evaluación previa que hizo la tutora sobre el tema de "la familia" relacionado con el centro de interés ya trabajado (el carnaval), realizamos una rutina de pensamiento como evaluación inicial de: (a) los conocimientos previos que 
tienen para partir de su nivel real; (b) los intereses sobre esta temática (lo que quieren aprender); y evaluación final (qué han aprendido).

2- Los alumnos reciben una carta de unas "criaturas". Para cuidar a las criaturas, se comprometerán a cumplir unos objetivos ("contrato"). Los objetivos los dicen y asumen los alumnos (guiados por la maestra), firman el contrato y son conscientes de que la evaluación irá conforme a estos objetivos.

\section{Evaluación}

En cada actividad de aprendizaje haremos consciente al alumno de los ítems de evaluación (contrato) a tener en cuenta. En la tabla 1 se muestran los instrumentos de evaluación a utilizar:

Tabla 1.

Instrumentos y técnicas de evaluación utilizados (elaboración propia)

\begin{tabular}{|c|c|c|}
\hline TÉCNICAS & INSTRUMENTOS & DESARROLLO \\
\hline \multirow{2}{*}{$\begin{array}{l}\text { Observación } \\
\text { participante }\end{array}$} & $\begin{array}{l}\text {-Cuaderno del } \\
\text { profesor }\end{array}$ & $\begin{array}{l}\text { Usos: (1) anecdotario, para los aspectos más significativos durante y } \\
\text { tras cada actividad de aprendizaje; (2) para reflexionar sobre la } \\
\text { observación. }\end{array}$ \\
\hline & $\begin{array}{l}\text {-Fichas de } \\
\text { seguimiento grupal }\end{array}$ & $\begin{array}{l}\text { Durante la semana se observa y se da feedback a los alumnos. Al } \\
\text { finalizar la semana completa la ficha con las valoraciones de la } \\
\text { maestra. }\end{array}$ \\
\hline \multirow{3}{*}{$\begin{array}{l}\text { Evaluación } \\
\text { entre iguales }\end{array}$} & \multirow{3}{*}{$\begin{array}{l}\text {-Ficha de evaluación } \\
\text { entre iguales } \\
\text { (coevaluación) }\end{array}$} & En tres actividades de aprendizaje grupales (primera intervención). \\
\hline & & $\begin{array}{l}\text { Al comenzar la actividad, se pactan los ítems que tendremos en cuenta } \\
\text { para poner una pegatina verde (cumplir todos los ítems), azul (cumplir } \\
\text { alguno) o ninguna (no cumplir ninguno). Un portavoz por grupo } \\
\text { (elegido por la profesora) explica lo que ha realizado. Los demás } \\
\text { comentan si se merece pegatina azul o verde, o ninguna, por qué, y } \\
\text { después comento lo que he escrito en mi cuaderno del profesor. }\end{array}$ \\
\hline & & Los alumnos co-evalúan a cada compañero (segunda intervención). \\
\hline $\begin{array}{l}\text { Reflexión } \\
\text { docente }\end{array}$ & -Diario del profesor & $\begin{array}{l}\text { Para reflexionar sobre: (1) aprendizajes de los alumnos; (2) mejoras en } \\
\text { la práctica docente; (3) aspectos positivos práctica docente. }\end{array}$ \\
\hline \multirow{3}{*}{$\begin{array}{l}\text { Evaluación } \\
\text { compartida }\end{array}$} & & $\begin{array}{l}\text {-Como evaluación del proceso de E-A se usa la técnica: "el tendero de } \\
\text { los deseos" (García-Herranz, 2017). }\end{array}$ \\
\hline & $\begin{array}{l}\text {-Rúbrica de } \\
\text { aprendizaje }\end{array}$ & \multirow{2}{*}{$\begin{array}{l}\text {-La maestra completa una rúbrica de aprendizaje sobre la evolución de } \\
\text { cada alumno. Los ítems están directamente relacionados con los } \\
\text { objetivos/compromisos fijados por los alumnos. La maestra realiza la } \\
\text { evaluación de cada alumno; después pasa a la evaluación compartida } \\
\text { (conversación individual con cada alumno sobre su trabajo en dichos } \\
\text { criterios). La maestra ofrece feedback y argumentos objetivos a lo que } \\
\text { el alumno dice. }\end{array}$} \\
\hline & $\begin{array}{l}\text {-Escala graduada } \\
\text { para la } \\
\text { autoevaluación }\end{array}$ & \\
\hline
\end{tabular}

La evaluación formativa y compartida en Educación Infantil. Consecuencias del uso de dos metodologías 
La Evaluación Compartida se realizará:

1- "El tendero de los deseos" (García-Herranz, 2017) es un instrumento para “conseguir información lo más precisa y objetiva posible del proceso de E-A desarrollado" (García-Herranz y López-Pastor, 2015, p. 2084). Cada alumno dibuja o escribe en una hoja de color verde lo que mas les ha gustado y/o en una hoja de color roja lo que menos. Después colocan en una cuerda sus "deseos" y explicando lo que ha querido transmitir en el papel. De esta manera obtenemos una evaluación consensuada del alumnado sobre su propio proceso de enseñanza-aprendizaje (E-A), buscaremos alternativas sobre lo que no funciona y mantendremos lo que ha funcionado.

2-Entrevistas dialogadas (véase tabla 1). Sirve como autoevaluación individual con cada alumno. Se realiza al finalizar la segunda intervención, englobando todo lo trabajado.

\section{Conclusiones}

Las conclusiones se mostrarán en el congreso de Evaluación Formativa y Compartida (Segovia, 07-2019). Actualmente podemos aportar los datos de la primera intervención:

-Los diálogos grupales son cada vez más fluidos. La participación y relación con los criterios de evaluación va mejorando progresivamente.

-Hay una evolución progresiva y positiva en las coevaluaciones. Cuanto más se realizan estas rutinas, más reflexivos son sus comentarios.

-Rechazo ante la creación de objetivos. La rutina de pensamiento fue un trabajo costoso para los alumnos. No se invertirá tanto tiempo en próximas ocasiones.

-Respeto y cumplimiento de los criterios de evaluación. Los alumnos se muestran muy justos ante la evaluación de actividades.

-Existe cierta imitación en las autoevaluaciones sobre lo que se ha aprendido (rutina de pensamiento). Al ser la primera autoevaluación que hacen, dicen lo primero que escuchan.

-Del recurso del "tendero de los deseos" surgió una evaluación muy objetiva del proceso de E-A.

-Recopilar las intervenciones de los alumnos en el cuaderno del profesor es un trabajo muy útil, pero muy costoso. Pensaremos cómo hacerlo de manera más eficaz. 


\section{Referencias}

Álvarez-Méndez, J. M. (2001). Evaluar para conocer, examinar para excluir. Madrid: Morata.

García-Herranz, S. (2017). Una experiencia de estimulación temprana y evaluación formativa en educación infantil. En López-Pastor \& Pérez-Pueyo (Ed.). Buenas Prácticas Docentes (pp. 118-126). León: Grupo IFAHE.

García-Herranz, S., \& López-Pastor, V. M. (2015). Evaluación Formativa y Compartida en Educación Infantil. Revisión de una Experiencia Didáctica. Qualitative Research in Education, 4(3), 269-298. doi:10.17583/qre.2015.1269

López-Pastor, V. M. \& Pérez-Pueyo, A. (coords.) (2017). Evaluación formativa y compartida en educación: experiencias de éxito en todas las etapas educativas. León: Universidad de León. (e-book). Recuperado de https://buleria.unileon.es/handle/10612/5999 